\title{
Bilateral neck exploration in patients with primary hyperparathyroidism and discordant imaging results: a single-centre study
}

\author{
Melanie Philippon ${ }^{1}$, Carole Guerin ${ }^{2}$, David Taieb ${ }^{3}$, Josiane Vaillant ${ }^{4}$, \\ Isabelle Morange ${ }^{1}$, Thierry Brue ${ }^{1}$, Bernard Conte-Devolx ${ }^{1}$, Jean-Franois Henry ${ }^{2}$, \\ Evelyne Slotema ${ }^{2}$, Frederic Sebag ${ }^{2,+}$ and Frederic Castinetti ${ }^{1,+}$ \\ Departments of ${ }^{1}$ Endocrinology, ${ }^{2}$ Endocrine Surgery, ${ }^{3}$ Nuclear Medicine and ${ }^{4}$ Radiology, Aix Marseille University, \\ La Timone Hospital, Marseille, 13005 France \\ ${ }^{\dagger}$ (F Sebag and F Castinetti contributed equally to this work)
}

\author{
Correspondence \\ should be addressed \\ to F Castinetti \\ Email \\ Frederic.castinetti@ap-hm.fr
}

\begin{abstract}
Introduction: Focused parathyroidectomy is the treatment of choice for patients with concordant positive imaging.

Bilateral cervical exploration is performed for cases with discordant imaging, yet more than $70 \%$ of those cases are the result of a single-gland disease. As focused parathyroidectomy is generally costless and harmless, for cases with discordant imaging, we tried to determine whether preoperative characteristics can lead to a diagnosis of single-gland disease. Methods: This study included 182 patients treated for primary hyperparathyroidism by bilateral exploration from 2009 to 2012 at La Timone Hospital, Marseille, France. We classified patients based on preoperative images and pathological results (single-gland or multiglandular disease). We then compared the demographical, laboratory and imaging results. We also asked a senior nuclear medicine practitioner who was blind to the ultrasound and pathological results to perform a second reading.
\end{abstract}

Results: Of the total number of patients, $15.4 \%$ had negative, $54.4 \%$ discordant and $30.2 \%$ concordant imaging.

After reviewing the scintigraphy results, $8 \%$ of the cases with discordant imaging would have been classified as concordant with ultrasound. Subtraction scintigraphy obtained better results than dual-phase scintigraphy (concordance with ultrasound in 50 vs $31 \%$ with classical scintigraphy). For the cases of discordant imaging, no predictive factors of single-gland disease could be identified. Ultrasound and scintigraphy were similarly effective in determining the correct location of the abnormal gland. Conclusion: Discordant results of preoperative imaging modalities do not discriminate between uniglandular and multiglandular diseases in hyperparathyroidism. Diagnostic differentiation between the different causes of hyperparathyroidism requires improvements in imaging techniques and might benefit from subtraction scintigraphy.

\section{Introduction}

Primary hyperparathyroidism (PHPT) is the first aetiology of hypercalcaemia and the third most frequently occurring endocrine disease. Its prevalence is estimated to be $0.1-2 \%$, while its incidence has increased over recent years up to $30 / 100000$ per year. PHPT rarely leads to severe hypercalcaemia; it is now frequently diagnosed at an asymptomatic stage and less frequently because of recurring kidney stones or osteoporosis. Thus, PHPT is, in the large majority of cases, a benign disease that is responsible for increased morbidity, and it requires an optimal and harmless management strategy $(1,2,3)$.

PHPT is the result of a single adenoma in $80-85 \%$ cases. Initial imaging workouts, i.e. ultrasound and ${ }^{99} \mathrm{~m}$ Tc-sestamibi scintigraphy, are aimed at determining
(C) 2014 European Society of Endocrinology Printed in Great Britain
Published by Bioscientifica Ltd. 
the uniglandular or multiglandular nature of the disease to allow focused parathyroidectomy $(4,5)$, the preferred treatment for cases of single adenoma. In contrast, multiglandular disease (adenoma or hyperplasia), which has been reported in $15-20 \%$ cases, requires bilateral exploration $(1,4,5,6)$. Imaging sensitivity depends on the nature of the disease: up to 91 vs $30-45 \%$ for scintigraphy $(4,7,8,9)$ and $77-80$ vs $16-35 \%$ for ultrasound in single-gland vs multiglandular disease respectively $(9,10)$. In contrast, the specificity for both procedures is $91-98 \%(11,12,13)$. Moreover, the success of ultrasound mainly depends on the radiologist's experience (14). Data in the literature showed that up to $89 \%$ of cases of single adenoma could be individualised using both techniques (15). However, $24-27 \%$ of preoperative imaging results are discordant $(6,16)$ and $12-18 \%$ are negative $(17,18,19)$; for these cases, the gold standard for surgery remains bilateral exploration (5). The choice between the treatment strategies of focused parathyroidectomy and bilateral exploration remains a matter of debate $(20,21,22,23)$. However, recent data in the literature suggested that focused parathyroidectomy was less expensive and induced fewer side effects with at least identical remission rates, resulting in a shorter hospital stay $(5,24)$. Increasing the number of patients treated by focused parathyroidectomy should be the main objective of pre-surgical imaging workouts.

We decided to focus on patients with PHPT with discordant imaging. As bilateral exploration has previously been used for all patients with negative or discordant imaging at our centre, we retrospectively evaluated all the patients with bilateral exploration between 2009 and 2012. Our main objective was to determine whether clinical, laboratory or preoperative imaging results could have guided the surgeon to the identification of a uniglandular disease resulting in a less invasive surgery, despite the presence of discordant or negative imaging.

\section{Subjects and methods}

All patients treated for PHPT by bilateral exploration in the Department of Endocrine Surgery of La Timone Hospital, Marseille, France, between May 2009 and June 2012 were retrieved from a local database. This selection criterion was justified by the systematic bilateral neck exploration in case of discordant or negative imaging (ultrasound and scintigraphy) in our department. We included only patients who had their initial imaging workout at our hospital, including ultrasound performed by a radiologist specialised in thyroid and parathyroid ultrasound
(J Vaillant) and a ${ }^{99 \mathrm{~m}} \mathrm{Tc}$-sestamibi scintigraphy performed by a senior nuclear medicine practitioner. All patients signed a consent allowing the retrospective study to be performed, as required by the Ethics Committee of our institution.

PHPT was defined by hypercalcaemia associated with a non-decreased parathormone (PTH) level. Blood samples were obtained and analysed systematically at the Department of Biology at La Timone Hospital. The laboratory normal values were: plasma calcium level, 2.25-2.58 mmol/l; phosphorus level, $0.79-1.53 \mathrm{mmol} / \mathrm{l}$; PTH level, 15.1-65.1 pg/ml and vitamin D level, $75-250 \mathrm{nmol} / \mathrm{l}$. Calcium level was corrected when albumin level was $<38 \mathrm{~g} / \mathrm{l}$.

For each patient, demographic (sex and age at diagnosis), laboratory (plasma calcium, phosphorus, PTH, vitamin D, urea, creatinine and urinary calcium levels), genetic (menin (MEN1), RET and HRPT2 (CDC73), when performed) and imaging results (ultrasound and scintigraphy) were recorded. Ultrasound and scintigraphy were considered to be concordant when the precise location (side and upper or lower position) and the exact number of glands considered abnormal were similar. Preoperative (number of glands resected) as well as postsurgical laboratory data (plasma calcium and PTH) were also recorded. The pathology data included the weight of the glands (mean in case of multiglandular disease) and the number and nature of the pathological gland(s) (adenoma or hyperplasia). The patient was considered to be cured when the post-operative plasma calcium level was low or normal and all four glands had been visualised during the surgery or when the intraoperative PTH level had decreased $(>50 \%)$ in case of incomplete visualisation.

To better characterise this cohort, we first classified patients into three groups based on their imaging results: group 1 with positive concordant imaging (including one or several abnormal glands), group 2 with discordant imaging (with only one positive image or both positive but with different results) and group 3 with negative imaging. We then compared the demographic and laboratory characteristics and pathological findings in each group.

As a second step, we classified patients into two groups based on the uni- or multiglandular nature of their disease determined by the pathological findings. Parathyroid disease could be classified as adenoma or hyperplasic gland. Patients who were not cured after surgery despite a single pathological gland resection were finally classified as having multiglandular disease. We compared the demographic, laboratory and imaging characteristics between these two groups to determine whether some 


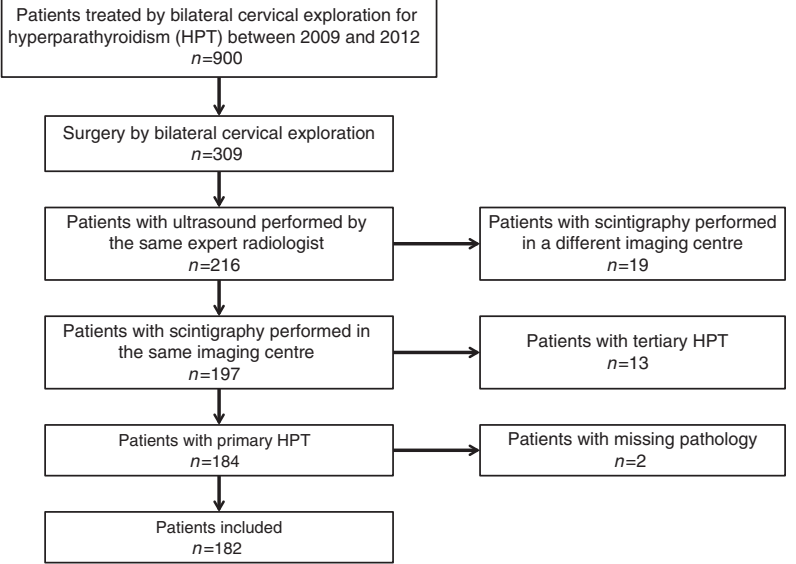

Figure 1

Flow chart summarising the characteristics of patients included.

pre-surgical parameters could have guided the clinician to the diagnosis of a single-gland disease when the imaging results were discordant. In the uniglandular disease group with discordant preoperative imaging, we also compared the performance of ultrasound and scintigraphy (divided into two groups, dual-phase sestamibi vs iodine-123technetium-99m-sestamibi subtraction scintigraphy) for defining the correct location of the pathological gland. Finally, we asked a second senior physician of the Department of Nuclear Medicine (D Taieb), specialised in endocrine disease and blind to the results of the ultrasound and the final pathological result, to read all the scintigraphies, which had been originally performed by a different nuclear medicine practitioner.

Statistics were performed with XLStat Software (Adinsoft, Paris, France). The quantitative data are presented as the means \pm s.D. The qualitative data were compared by the $\chi^{2}$ or Fisher's exact test. The quantitative data were compared by ANOVA or Student's $t$-test. A $P$ value of $<0.05$ was considered significant.

\section{Results}

In this retrospective study, 182 patients, 131 females and 51 males (mean age at diagnosis: $62.8 \pm 13.5$ years), were included (Fig. 1). The demographic and laboratory preand post-surgical data are summarised in Table 1 . The median follow-up was 0.3 month (min., 0.3 and max., 36). At last follow-up, 174 patients (95.6\%) were cured. A total of eight patients were uncured. Their main characteristics are available in Supplementary Table 1, see section on supplementary data given at the end of this article.

\section{Classification based on imaging results}

A total of 55 patients (30.2\%) had both positive and concordant ultrasound and scintigraphy imaging (group 1). Bilateral cervical exploration was justified because of the need for thyroid resection in 27 patients (49\%), imaging showing several abnormal glands in 16 patients (29.1\%), the mediastinal or ectopic location of the parathyroid gland in four patients $(7.3 \%)$ or recurrence in eight patients $(14.5 \%)$. Of the total number of patients, 99 (54.4\%) had discordant imaging (group 2), and 28 patients (15.4\%) had negative imaging for both ultrasound and scintigraphy (group 3) (Fig. 2).

Pre-surgical characteristics - The demographic (age at diagnosis and sex ratio) and laboratory data (plasma calcium, PTH and vitamin D levels) were not significantly different among the three groups. Additionally, there was no significant difference in the number of bilateral explorations due to thyroid resection between the three groups $(P=0.66)$.

The imaging workouts included 138 (76\%) dual-phase sestamibi scintigraphies and 44 (24\%) subtraction scintigraphies.

Patients with positive concordant imaging underwent subtraction scintigraphy (32.7\% of cases) more frequently than patients with discordant or negative imaging (21.2 and $17.8 \%$ of cases respectively) $(P=0.02)$. Concordance with the pathology results was more frequent with subtraction scintigraphy than with classical sestamibi scintigraphy (50 vs $31 \%, P=0.02$ ). For patients with discordant imaging, there was no significant difference between scintigraphy and ultrasound in determining the

Table 1 Main characteristics of the whole population $(n=182)$. Data are presented as mean \pm s.D.

\begin{tabular}{lcc}
\hline Characteristics & & Values \\
\cline { 1 - 1 } Preoperative data & & \\
Sex ratio (female:male) & & $131: 51(72 \%: 28 \%)$ \\
Age at diagnosis (years) & $62.8 \pm 13.5$ \\
Calcium (mmol/l) & $2.74 \pm 0.2$ \\
Phosphorus (mmol/l) & $0.82 \pm 0.2$ \\
PTH (pg/ml) & $168.3 \pm 177.5$ \\
Vitamin D (nmol/l) & $59.4 \pm 33.6$ \\
Creatinine ( $\mu \mathrm{mol} / \mathrm{l})$ & $74.9 \pm 42.9$ \\
Postoperative data & \\
PTH D0 $+4 \mathrm{~h}(\mathrm{pg} / \mathrm{ml})$ & $20.3 \pm 43.9$ \\
PTH D1 (pg/ml) & $27.4 \pm 48.3$ \\
PTH D8 (pg/ml) & $49.5 \pm 47.4$ \\
Calcium D1 (mmol/l) & $2.21 \pm 0.17$ \\
Calcium D8 (mmol/l) & $2.27 \pm 0.17$ \\
Follow-up (months) & $3.03 \pm 7.4$ \\
\hline
\end{tabular}

PTH, parathormone; D0, day of surgery; D1, first post-operative day; D8, eighth postoperative day. 


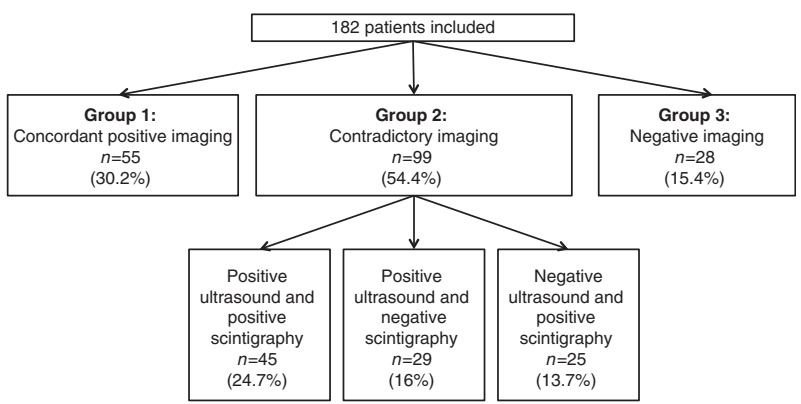

Figure 2

Imaging characteristics of patients included.

correct location of the abnormal gland(s) (43.5 vs $38.5 \%$ respectively; $P=0.43$ ).

A second reading by a specialised nuclear medicine practitioner allowed 9\% (9/99) of discordant scintigraphy cases to be classified as concordant with ultrasound. If a second reading had been performed before surgery, $8 \%$ of discordant scintigraphy cases would have been considered as positive concordant imaging in favour of single-gland disease, leading to focused parathyroidectomy.

Pathology - There was no significant difference in the rate of multiglandular disease among the three groups $(29,30.5$ and $28 \%$ in groups 1,2 and 3 respectively; $P=0.717$ ). The mean gland weight was significantly lower in patients with negative imaging for both ultrasound and scintigraphy when compared with patients with concordant positive imaging $(0.39 \pm 0.32$ vs $1.22 \pm 1.13 \mathrm{~g} ; P=0.04)$. There was no significant difference when comparing patients with negative or positive imaging and those with discordant imaging (mean weight: $0.984 \pm 1.03$ g; Table 2 ).

Post-surgical data - The remission rates were similar among the three groups $(94.5,94.1$ and $96.4 \%$ for groups 1,2 and 3 respectively; $P=0.98$ ). The mean follow-up was not significantly different between the three groups $(0.83 \pm 2.75,3.99 \pm 8.45$ and $3.99 \pm 8.16$ months, for groups 1,2 and 3 respectively; $P=0.138$ ).

\section{Classification based on single-gland vs multiglandular disease}

Pathological results of resected glands were normal for nine patients (4.9\%). Of the 173 remaining patients, 122 (70.5\%) had a single-gland disease, whereas $51(29.5 \%)$ had a multiglandular disease. In the singlegland disease group, the pathological analysis revealed an adenoma in $83 \%$ and a hyperplasia in $17 \%$ of cases. In the multiglandular disease group, 55.5\% had two abnormal glands, $28.9 \%$ had three abnormal glands and $15.5 \%$ had four abnormal glands.

Post-surgical data $>$ The gland weight was not significantly different between the two groups (single-gland vs multiglandular disease; $P=0.08$ ). Patients with multiglandular disease more frequently had a calcium level $<2.2 \mathrm{mmol} / \mathrm{l}$ (37.5 vs 19.2\%; $P=0.018$ ) at day 8 . All the uncured patients $(n=8)$ presented with multiglandular disease.

Pre-surgical predictive factors of single-gland disease We tried to determine retrospectively whether some preoperative predictive factors could have guided the surgeon to the diagnosis of a single-gland disease in case of discordant imaging.

There was no significant difference in terms of demographic (age at diagnosis and sex) or laboratory data, including plasma calcium, phosphorus and creatinine levels, between the two groups. In the univariate analysis, the pre-surgical PTH level was significantly higher in the multiglandular vs the single-gland disease groups (mean: $220 \pm 238$ vs $152 \pm 143.5 \mathrm{pg} / \mathrm{ml} ; P=0.02$ ). However, the vitamin D level was also significantly

Table 2 Main preoperative and pathological results for the three imaging groups of patients.

\begin{tabular}{lcc}
\hline & & $\begin{array}{c}\text { Patients with } \\
\text { concordant imaging }\end{array}$ \\
\hline Calcium (mmol/l) (mean \pm s.D.) & & $2.75( \pm 0.25)$ \\
PTH preoperative level (pg/ml) (mean \pm s.D.) & & $27 / 55(49 \%)^{*}$ \\
Thyroid pathology (patients) & & $1.22( \pm 1.13) *$ \\
Weight of pathological gland (g) (mean \pm s.D.) & & $39 / 55(71 \%)$ \\
Uniglandular disease (patients) & $16 / 55(29 \%)$ \\
Multiglandular disease (patients) &
\end{tabular}

\begin{tabular}{c} 
Patients with \\
discordant imaging \\
\hline $2.73( \pm 0.17)$ \\
$152( \pm 142)$ \\
$30 / 99(30.3 \%)$ \\
$0.984( \pm 1.03)$ \\
$69 / 99(69.5 \%)$ \\
$30 / 99(30.5 \%)$ \\
\hline
\end{tabular}

\begin{tabular}{c}
$\begin{array}{c}\text { Patients with } \\
\text { negative imaging }\end{array}$ \\
\hline $2.72( \pm 0.14)$ \\
$126( \pm 124.1)$ \\
$5 / 28(17.8 \%)$ \\
$0.39( \pm 0.32) *$ \\
$20 / 28(72 \%)$ \\
$8 / 28(28 \%)$
\end{tabular}

\begin{tabular}{l}
\hline \multicolumn{1}{c}{$\boldsymbol{P}$} \\
\hline 0.57 \\
0.47 \\
0.04 \\
$0.04^{\star}$ \\
0.72 \\
0.72
\end{tabular}

Significant result $(* P<0.05)$ between these two groups of patients 
Table 3 Comparative characteristics of patients with single-gland vs multiglandular disease. Data are presented as mean \pm s.D.

\begin{tabular}{|c|c|c|c|}
\hline & $\begin{array}{c}\text { Patients with } \\
\text { single gland disease }\end{array}$ & $\begin{array}{c}\text { Patients with } \\
\text { multiglandular disease }\end{array}$ & $\boldsymbol{P}$ \\
\hline Age at diagnosis (years) & $63.88 \pm 13$ & $61.93 \pm 14.1$ & 0.38 \\
\hline Calcium level $(\mathrm{mmol} / \mathrm{l})$ & $2.75 \pm 0.19$ & $2.75 \pm 0.22$ & 0.94 \\
\hline PTH (pg/ml) & $151.97 \pm 143.53$ & $219.75 \pm 238.01$ & 0.02 \\
\hline Vitamin D (nmol/l) & $64.01 \pm 36.86$ & $49.60 \pm 24.14$ & 0.01 \\
\hline PTH level at D0 $+4 \mathrm{~h}(\mathrm{pg} / \mathrm{ml})$ & $13.65 \pm 9.59$ & $30.57 \pm 75.6$ & 0.02 \\
\hline PTH level at D1 (pg/ml) & $20.36 \pm 14.98$ & $38.94 \pm 83.77$ & 0.02 \\
\hline PTH level at D8 $(\mathrm{pg} / \mathrm{ml})$ & $42.61 \pm 22.38$ & $64 \pm 77.29$ & 0.01 \\
\hline
\end{tabular}

$P<0.05$ was considered significant. PTH, parathormone; D0, day of surgery; D1, first postoperative day; D8, eighth postoperative day.

different between the two groups (mean: $50 \pm 24.1$ vs $64 \pm$ $36.8 \mathrm{nmol} / \mathrm{l} ; P=0.01$; Table 3 ).

The imaging results were better in cases of uniglandular disease: $47 \%$ of ultrasound results and $44.4 \%$ of scintigraphy results were concordant with the pathological findings in the single-gland disease group compared with only $14 \%$ in the multiglandular disease group $(P<0.0001)$.

\section{Discussion}

Our results showed a similar proportion of multiglandular disease in both negative and discordant imaging: multiglandular disease was observed in $28-30 \%$ of patients, which means that $70 \%$ of the cohort had a single-gland disease. Surprisingly, the rate of multiglandular disease was not significantly different among these two groups and the group of patients with concordant positive imaging. However, this latter group was biased by the inclusion of patients with several concordant images and the exclusion of patients treated by focused parathyroidectomy because of a single positive and concordant image ( $n=590$ in the same period). Thus, our data do not contradict previously published data, which showed that multiglandular disease was more frequent in case of discordant or negative imaging $(19,25,26)$.

We did not identify pre-surgical factors of single-gland disease other than imaging. The only significant parameter was the level of PTH, which was higher in the multiglandular disease group. However, this was probably explained by lower levels of vitamin D. Discordant results have been reported in the literature: Thakur et al. (27) did not show any difference in PTH or calcium levels in cases of single-gland and multiglandular diseases. In contrast, Kebebew et al. (26) created a score that included calcium, PTH and imaging results allowing for the detection of uniglandular disease with a $100 \%$ positive predictive value and specificity. On one hand, using this score for our patients with discordant imaging would have led to a false conclusion of single-gland disease in $26.7 \%$ of cases; on the other hand, it would have clearly increased the numbers of appropriate surgical approaches, i.e. the rate of focused parathyroidectomy in case of single-gland disease. The difference in the predictive value of this score in our study could be explained by our selection criteria; we used this score to assess a more challenging population, which theoretically represents only onefourth of all patients with hyperparathyroidism.

When imaging was discordant, none of the imaging was superior in localising the abnormal gland, in contrast with previous literature data $(25,28)$. Our results suggest that the use of systematic subtraction scintigraphy and a second reading by a specialised nuclear medicine practitioner added to the pre-surgical score would clearly increase the rate of focused parathyroidectomy in patients with discordant imaging. However, literature data showed discordant results, with a better sensitivity, but a lower predictive positive value for subtraction vs classical scintigraphy $(11,29)$. This point is difficult to consider in our patients due to the initial selection bias in our cohort. A second reading of the scintigraphy by a specialised nuclear medicine practitioner increased the rate of concordant positive imaging with ultrasound (9\% increase). This had already been shown for ultrasound; a second ultrasound imaging reading allowed $65 \%$ of discordant imaging results to be reclassified as concordant (16). Of note, the facts that ultrasound was performed by a specialised radiologist and that we included only the scintigraphies read by a senior physician of the Department of Nuclear Medicine probably explain why the rates of discordant imaging were lower than those in the literature (6).

Finally, performance of two preoperative imaging procedures (ultrasound and scintigraphy) remains theoretically debatable when the imaging is performed under optimal conditions and intraoperative PTH assay is 
easily available (30): for instance, Kunstman et al. (5) recommended focused parathyroidectomy when the first imaging was clearly considered positive. In our patients with only one positive and formal imaging, the application of this management approach would have led to appropriate focused parathyroidectomy in 43\% of our patients (and surgical conversion in enlarged cervicotomy in 57\%; data not shown).

Our study has several limitations. As our main objective was to understand how to improve the management of patients with discordant imaging, we did not analyse the data of the 590 patients, with PHPT and concordant imaging, who underwent focused parathyroidectomy in the same period of time. The main advantage of our selection was that we had two subgroups of control patients: positive concordant with several images and negative concordant (i.e. negative imaging for both ultrasound and scintigraphy). The originality of this approach, however, makes it difficult to compare with previously published results, which included a majority of 'obvious' cases. Another limitation was the time between surgery and the determination of the calcium level used to define remission (8 days). As to whether or not this could hinder the identification of recurrence in patients who underwent focused parathyroidectomy, we believe that patients with bilateral exploration are less at risk of developing recurrence, as all of their glands would theoretically be explored, or as they would have had preoperative PTH samplings (data not shown). Finally, we tried to be as strict as possible in the definition of concordance. We considered images to be concordant only if they showed the same side and upper/lower location of the abnormal gland(s), while the majority of previously published studies used only the side of the gland in their definition of concordance. This strict criterion was used to ensure that only patients with absolute imaging certitude of a single-gland disease would be considered appropriate for focused parathyroidectomy.

To conclude, discordant or negative imaging does not necessarily mean multiglandular disease, as 70\% of our patients had uniglandular disease. Therefore, these patients would clearly benefit from focused parathyroidectomy. Our study did not manage to identify any predictive factors other than imaging that could help distinguish between single-gland and multiglandular diseases. The only ways to improve the pre-surgical workout are based on a second reading by an expert nuclear medicine practitioner and the systematic use of subtraction scintigraphy. Taking into account the higher cost and morbidity of bilateral exploration, we believe that our results emphasise the need for the management of PHPT in specialised centres including expert radiologists and nuclear medicine practitioners.

\section{Supplementary data}

This is linked to the online version of the paper at http://dx.doi.org/10.1530/ EJE-13-0796.

\section{Declaration of interest}

The authors declare that there is no conflict of interest that could be perceived as prejudicing the impartiality of the research reported.

\section{Funding}

This research did not receive any specific grant from any funding agency in the public, commercial or not-for-profit sector.

\section{References}

1 Fraser WD. Hyperparathyroidism. Lancet 2009374 145-158. (doi:10.1016/S0140-6736(09)60507-9)

2 Langdahl BL \& Ralston SH. Diagnosis and management of primary hyperparathyroidism in Europe. QJM: Monthly Journal of the Association of Physicians 2012105 519-525. (doi:10.1093/qjmed/hcr225)

3 Pallan S \& Khan A. Primary hyperparathyroidism: update on presentation, diagnosis, and management in primary care. Canadian Family Physician Médecin de Famille Canadien 201157 184-189.

4 Udelsman R, Pasieka JL, Sturgeon C, Young JEM \& Clark OH. Surgery for asymptomatic primary hyperparathyroidism: proceedings of the third international workshop. Journal of Clinical Endocrinology and Metabolism 200994 366-372. (doi:10.1210/jc.2008-1761)

5 Kunstman JW, Kirsch JD, Mahajan A \& Udelsman R. Clinical review: Parathyroid localization and implications for clinical management. Journal of Clinical Endocrinology and Metabolism $201398902-912$. (doi:10.1210/jc.2012-3168)

6 Nehs MA, Ruan DT, Gawande AA, Moore FD Jr \& Cho NL. Bilateral neck exploration decreases operative time compared to minimally invasive parathyroidectomy in patients with discordant imaging. World Journal of Surgery 201337 1614-1617. (doi:10.1007/s00268-013-2007-8)

7 Pappu S, Donovan P, Cheng D \& Udelsman R. Sestamibi scans are not all created equally. Archives of Surgery 2005140 383-386. (doi:10.1001/ archsurg.140.4.383)

8 Katz SC, Wang GJ, Kramer EL \& Roses DF. Limitations of technetium 99m sestamibi scintigraphic localization for primary hyperparathyroidism associated with multiglandular disease. American Surgeon 2003 69 170-175.

9 Ruda JM, Hollenbeak CS \& Stack BC Jr. A systematic review of the diagnosis and treatment of primary hyperparathyroidism from 1995 to 2003. Otolaryngology - Head and Neck Surgery 2005132 359-372. (doi:10.1016/j.otohns.2004.10.005)

10 Cheung K, Wang TS, Farrokhyar F, Roman SA \& Sosa JA. A metaanalysis of preoperative localization techniques for patients with primary hyperparathyroidism. Annals of Surgical Oncology 201219 577-583. (doi:10.1245/s10434-011-1870-5)

11 Lavely WC, Goetze S, Friedman KP, Leal JP, Zhang Z, Garret-Mayer E, Dackiw AP, Tufano RP, Zeiger MA \& Ziessman HA. Comparison of SPECT/CT, SPECT, and planar imaging with single- and dual-phase (99m)Tc-sestamibi parathyroid scintigraphy. Journal of Nuclear Medicine 200748 1084-1089. (doi:10.2967/jnumed.107.040428) 
12 Carlier T, Oudoux A, Mirallié E, Seret A, Daumy I, Leux C, BodetMilin C, Kraeber-Bodéré F \& Ansquer C. 99mTc-MIBI pinhole SPECT in primary hyperparathyroidism: comparison with conventional SPECT, planar scintigraphy and ultrasonography. European Journal of Nuclear Medicine and Molecular Imaging 200835 637-643. (doi:10.1007/ s00259-007-0625-9)

13 Tublin ME, Pryma DA, Yim JH, Ogilvie JB, Mountz JM, Bencherif B \& Carty SE. Localization of parathyroid adenomas by sonography and technetium tc $99 \mathrm{~m}$ sestamibi single-photon emission computed tomography before minimally invasive parathyroidectomy: are both studies really needed? Journal of Ultrasound in Medicine $2009 \mathbf{2 8}$ 183-190.

14 Van Husen R \& Kim LT. Accuracy of surgeon-performed ultrasound in parathyroid localization. World Journal of Surgery 200428 1122-1126. (doi:10.1007/s00268-004-7485-2)

15 Gayed IW, Kim EE, Broussard WF, Evans D, Lee J, Broemeling LD, Ochoa BB, Moxley DM, Erwin WD \& Podoloff DA. The value of 99mTc-sestamibi SPECT/CT over conventional SPECT in the evaluation of parathyroid adenomas or hyperplasia. Journal of Nuclear Medicine $200546248-252$

16 Chandramohan A, Sathyakumar K, Irodi A, Abraham D \& Paul MJ. Causes of discordant or negative ultrasound of parathyroid glands in treatment naïve patients with primary hyperparathyroidism. European Journal of Radiology 201281 3956-3964. (doi:10.1016/j.ejrad. 2012.08.019)

17 Chan RK, Ruan DT, Gawande AA \& Moore FD Jr. Surgery for hyperparathyroidism in image-negative patients. Archives of Surgery 2008143 335-337. (doi:10.1001/archsurg.143.4.335)

$18 \mathrm{Lal} \mathrm{A} \& \mathrm{Chen} \mathrm{H}$. The negative sestamibi scan: is a minimally invasive parathyroidectomy still possible? Annals of Surgical Oncology 200714 2363-2366. (doi:10.1245/s10434-007-9451-3)

19 Sebag F, Hubbard JGH, Maweja S, Misso C, Tardivet L \& Henry JF. Negative preoperative localization studies are highly predictive of multiglandular disease in sporadic primary hyperparathyroidism. Surgery 2003134 1038-1041 (discussion 1041-1042). (doi:10.1016/j. surg.2003.07.021)

20 Udelsman R. Six hundred fifty-six consecutive explorations for primary hyperparathyroidism. Annals of Surgery 2002235 665-670 (discussion 670-672). (doi:10.1097/00000658-200205000-00008)

21 Irvin GL III, Solorzano CC \& Carneiro DM. Quick intraoperative parathyroid hormone assay: surgical adjunct to allow limited parathyroidectomy, improve success rate, and predict outcome. World Journal of Surgery 200428 1287-1292. (doi:10.1007/s00268-004-7708-6)

22 Westerdahl J \& Bergenfelz A. Unilateral versus bilateral neck exploration for primary hyperparathyroidism: five-year follow-up of a randomized controlled trial. Annals of Surgery 2007246 976-980 (discussion 980-981). (doi:10.1097/SLA.0b013e31815c3ffd)

23 Russell CFJ, Dolan SJ \& Laird JD. Randomized clinical trial comparing scan-directed unilateral versus bilateral cervical exploration for primary hyperparathyroidism due to solitary adenoma. British Journal of Surgery 200693 418-421. (doi:10.1002/bjs.5250)

24 Udelsman R, Lin Z \& Donovan P. The superiority of minimally invasive parathyroidectomy based on 1650 consecutive patients with primary hyperparathyroidism. Annals of Surgery 2011253 585-591. (doi:10.1097/SLA.0b013e318208fed9)

25 Siperstein A, Berber E, Mackey R, Alghoul M, Wagner K \& Milas M. Prospective evaluation of sestamibi scan, ultrasonography, and rapid PTH to predict the success of limited exploration for sporadic primary hyperparathyroidism. Surgery 2004136 872-880. (doi:10.1016/j.surg. 2004.06.024)

26 Kebebew E, Hwang J, Reiff E, Duh QY \& Clark OH. Predictors of singlegland vs multigland parathyroid disease in primary hyperparathyroidism: a simple and accurate scoring model. Archives of Surgery 2006141 777-782 (discussion 782). (doi:10.1001/archsurg.141.8.777)

27 Thakur A, Sebag F, Slotema E, Ippolito G, Taïeb D \& Henry JF. Significance of biochemical parameters in differentiating uniglandular from multiglandular disease and limiting use of intraoperative parathormone assay. World Journal of Surgery 200933 1219-1223. (doi:10.1007/s00268-009-0029-z)

28 Arici C, Cheah WK, Ituarte PH, Morita E, Lynch TC, Siperstein AE, Duh QY $\&$ Clark OH. Can localization studies be used to direct focused parathyroid operations? Surgery 2001129 720-729. (doi:10.1067/msy.2001.114556)

29 Jorna FH, Jager PL, Que TH, Lemstra C \& Plukker JTM. Value of 123I-subtraction and single-photon emission computed tomography in addition to planar 99mTc-MIBI scintigraphy before parathyroid surgery. Surgery Today 200737 1033-1041. (doi:10.1007/s00595-0073550-4)

30 Singh DN, Gupta SK, Chand G, Mishra A, Agarwal G, Verma AK, Mishra SK, Shukla M \& Agarwal A. Intra-operative parathyroid hormone kinetics and influencing factors with high baseline PTH: a prospective study. Clinical Endocrinology 2013 78 935-941. (doi:10.1111/cen.12067)

Received 29 September 2013

Revised version received 18 February 2014

Accepted 25 February 2014 